\title{
Assessing Symptom Burden in Bladder Cancer: An Overview of Bladder Cancer Specific Health-Related Quality of Life Instruments
}

\author{
Bernard J. Danna*, Michael J. Metcalfe, Erika L. Wood and Jay B. Shah \\ Department of Urology, MD Anderson Cancer Center, Houston, TX, USA
}

\begin{abstract}
.
Background: A key component to monitoring and investigating patient QOL is through patient reported health related quality of life (HRQOL) outcome measures. Many instruments have been used to assess HRQOL in bladder cancer and each instrument varies in its development, validation, the context of its usage in the literature and its applicability to certain disease states.

Objective: In this review, we sought to summarize how clinicians and researchers should most appropriately utilize the available HRQOL instruments for bladder cancer.

Methods: We performed a comprehensive literature search of each instrument used in bladder cancer, paying particular attention to the outcomes assessed. We used these outcomes to group the available instruments into categories best reflecting their optimal usage by stage of disease.

Results: We found 5 instruments specific to bladder cancer, of which 3 are validated. Only one of the instruments (the EORTC-QLQ-NMIBC24) was involved in a randomized, prospective validation study. The most heavily used instruments are the EORTC-QLQ-BLM30 for muscle-invasive disease and the FACT-Bl which is used across all disease states. Of the 5 available instruments, 4 are automatically administered with general instruments, while the BCI lacks modularity, and requires co-administration with a generalized instrument.

Conclusion: There are multiple strong instruments for use in gauging HRQOL in bladder cancer patients. We have divided these instruments into three categories which optimize their usage: instruments for use following NMIBC treatments (EORTCQLQ-NMIBC24), instruments for use following radical cystectomy (FACT-Bl-Cys and EORTC-QLQ-BLM30) and more inclusive instruments not limited by treatment modality (BCI and FACT-Bl).
\end{abstract}

Keywords: Urinary bladder neoplasms/surgery, patient outcome assessment, cystectomy, health surveys, quality of life, psychometrics, cystectomy/psychology, postoperative complications/psychology, treatment outcome, urinary diversion/psychology

\section{BACKGROUND}

In 2016, more than 74,000 new cases of bladder

*Correspondence to: Bernard J. Danna, Department of Urology, MD Anderson Cancer Center, 1515 Holcombe Boulevard, Unit 1373, Houston, TX 77030, USA. Tel.: +1 832978 1696; E-mail: bernard.j.danna@uth.tmc.edu. cancer will be diagnosed in the United States [1]. Each patient presents at a different stage of disease and has a unique course. $70 \%$ of patients present with localized non muscle-invasive bladder cancer 
(NMIBC) and encounter numerous endoscopic and intra-vesical interventions throughout the disease course to try to prevent recurrence and progression of the disease. For patients with high risk NMIBC, disease progression, treatment refractory disease, or muscle invasive bladder cancer (MIBC), radical cystectomy with pelvic lymph node dissection (RCLND) and urinary diversion (UD) is the curative approach. The course of therapy for many patients with bladder cancer can be long, and the burden of therapy can be great. Furthermore, bladder cancer is among the most expensive adult cancers to treat per patient [2]. With each intervention it is necessary for the clinician to weigh the risks and benefits of treatment options as well as the effects of treatment on health related quality of life. To that end, several tools have been developed to gauge the effectiveness of bladder cancer treatments, with the end goal being optimal interventions for each patient at each point in their treatment course.

While mortality is the primary outcome when determining the effectiveness of treatment options, other patient-centered factors should be considered. For instance, understanding and reporting on the patient experience is of equivalent importance. This is reflected in academia and even extends through to congress. Since the early 2000 s, quality improvement in healthcare has emphasized a patientcentered and evidence-based approach [3, 4]. At the same time, the value-based payment modifier of the Affordable Care Act has necessitated improved reporting of clinical outcomes, including those on the patient experience and health related quality of life (HRQOL) [5, 6]. As a final factor driving a recent surge in HRQOL research, the Food and Drug Administration (FDA) is requiring patient-reported outcome (PRO) data in all clinical trials [7]. PRO data is especially relevant for bladder cancer, given the symptom burden of frequent recurrences requiring multiple cystoscopic examinations, intravesical therapy, as well as systemic therapy and complex surgical interventions for muscle-invasive disease. Investigators have recognized the necessity of PRO data in studying bladder cancer, and have responded by developing a wide range of HRQOL tools. Appropriate application of each tool at each disease state is as important as the quality of the tool itself. The purpose of this review is to give clarity to each of the tools and describe its appropriate use.

In this study, we compare the HRQOL tools that are applied to bladder cancer patients. Because many of these tools, or instruments, exist, investigators must determine which instrument(s) should be used in which particular circumstance, as the validity and response can only be applied to the setting in which the tool was developed [8]. Although many articles have been written which review existing HRQOL studies and the instruments used therein, to our knowledge no attempt has been made to holistically compare the HRQOL instruments specific to bladder cancer [8-11]. The purpose of this article is to summarize the major HRQOL instruments used in bladder cancer, and to provide an outline for investigators to choose the instrument(s) which is/are most suitable for their research needs.

\section{HRQOL INSTRUMENT OVERVIEW}

HRQOL is defined as the value that a patient assigns to duration of life as modified by impairments, functional states, perceptions, and social opportunities, and that are influenced by disease, injury, treatment or policy [9]. In a more general sense, HRQOL measures a patient's satisfaction with his or her health and its impact on life. Many different instruments are available for gauging HRQOL. These instruments can be categorized into general instruments, cancer-specific instruments and bladder cancer specific instruments (BCSIs) as outlined in Table 1.

General instruments are a non-specific measure of a patient's overall health. Questions in a general instrument are worded to address problems ranging from physical functioning to mental health to pain, such as those questions seen in the commonly used 36 question short form health survey (SF-36) [12]. Although these instruments are among the most validated and responsive (Table 2), they often lack the specificity to gauge HRQOL in patients with a particular disease, as they miss unique components of the effects that a disease has on an individual's wellbeing [8, 10, 13]. Cancer-specific instruments like the functional assessment of cancer therapy - general (FACT-G) and the EORTC-QLQ-C30 are designed to be used for patients with any type of cancer $[14,15]$. They are more specific than general instruments, with domains that pertain to the systemic effects of cancer. For example, the $\mathrm{C} 30$ includes five functional scales, three symptom scales, and six single items (Table 1) [14]. This breadth of data, although vital to the big picture, is excluded when BCSIs are used in isolation.

BCSIs are specially designed to gauge the urinary, bowel, sexual and self-image related symptoms 
Table 1

Instrument classifications

\begin{tabular}{|c|c|c|c|c|}
\hline & Instrument type & Module & Domains & Domains scored individually? \\
\hline \multicolumn{5}{|l|}{ General Instruments } \\
\hline FACT-G ${ }^{15}$ & Cancer-Specific & N/A & Physical, socia/family, emotional, functional & Yes \\
\hline \multirow[t]{3}{*}{ EORTC-QLQ-C30 ${ }^{14}$} & Cancer-Specific & N/A & $\begin{array}{l}\text { Functional (physical/role/emotional/ } \\
\text { cognitive/social) }\end{array}$ & Yes \\
\hline & & & Symptom (fatigue, nausea, vomiting/pain) & \\
\hline & & & $\begin{array}{l}\text { Single items (dyspnea, insomnia, appetite, } \\
\text { constipation, diarrhea, finances) }\end{array}$ & \\
\hline \multicolumn{5}{|l|}{ Nonmuscle-Invasive Instrument } \\
\hline \multirow[t]{2}{*}{ EORTC-QLQ-NMIBC24 21} & BCSI & $\mathrm{C} 30$ & $\begin{array}{l}\text { Grouped (urinary symptoms, malaise, future } \\
\text { worries, bloating, sexual function, male } \\
\text { sexual problems) }\end{array}$ & No \\
\hline & & & $\begin{array}{l}\text { Single items (Intravesical treatment issues, } \\
\text { sexual intimacy, risk of contaminating } \\
\text { partner, sexual enjoyment, female sexual } \\
\text { problems) }\end{array}$ & \\
\hline \multicolumn{5}{|l|}{ Muscle-Invasive Instruments } \\
\hline EORTC-QLQ-BLM3020 & BCSI & $\mathrm{C} 30$ & $\begin{array}{l}\text { Single items (urinary symptoms, sexual } \\
\text { function, urostomy issues, catheter use, } \\
\text { body image) }\end{array}$ & No \\
\hline FACT-B1-Cys ${ }^{22}$ & BCSI & FACT-G & $\begin{array}{l}\text { Single items (urinary symptoms, sexual } \\
\text { interest, bowel function, body image, } \\
\text { appetite) }\end{array}$ & No \\
\hline \multicolumn{5}{|c|}{ Inclusive Bladder Cancer Instrument } \\
\hline $\mathrm{BCI}^{23}$ & BCSI & No & $\begin{array}{l}\text { Grouped (blader, bowel and sexual function } \\
\text { with function and bother subdomains for } \\
\text { each) }\end{array}$ & Yes \\
\hline FACT-B1 $1^{32}$ & BCSI & FACT-G & $\begin{array}{l}\text { Single items (urinary function, sexual } \\
\text { function, bowel function, appetite, ostomy } \\
\text { care, body image) }\end{array}$ & No \\
\hline
\end{tabular}

Note: Principle Component Analysis was completed to determine if questionnaire items should be grouped or single. Some questionnaires (the NMIBC24 and C30) have both grouped domains and single items. The BCI is the only BCSI which has individual scores for each domain. See definition of Principal Component Analysis in Appendix 1.

specific to bladder cancer and its treatments. There are many BCSI's; we describe 5 of the most known: the EORTC-QLQ-NMIBC24 (to compare between nonmuscle-invasive treatment modalities), the EORTC-QLQ-BLM30 and the FACT-Bl-Cys (to compare between muscle invasive treatment modalities), as well as the FACT-Bl and BCI (to compare across treatment modalities). If body image is a consideration, investigators should consider including the EORTC body image scale in conjunction with a BCSI [16]. Other important considerations include gender and choice of urinary diversion [8]. Although BCSIs are the gold standard for use in studying bladder cancer, they are often specific to the point of missing important information about a person's QOL, which can be collected by general instruments or cancer-specific instruments. Thus, all BCSIs should be co-administered with a more general instrument, like the FACT-G, EORTC-QLQ-C30, or SF-36.

Because of the smaller sample size of studies using BCSIs, they tend to be less well psychometrically vetted than their more established general counterparts [13]. At a minimum, all studies should be psychometrically evaluated in terms of acceptability, reliability, validity, responsiveness, and cultural adaptation [17]. A description of these metrics is available in the Appendix 1, and analysis of these metrics for each instrument is located in the instrument descriptions below.

In summary, an appropriate HRQOL instrument to study any particular disease must be well validated and responsive to the many domains of a patient's HRQOL. It must be general enough to assess overall well-being, and specific enough to assess the patient's disease and treatments. In the following section, each BCSI will be examined in detail.

\section{METHODS}

Our literature search focused on the individual instruments used to assess quality of life in patients with bladder cancer. To find out which instruments 


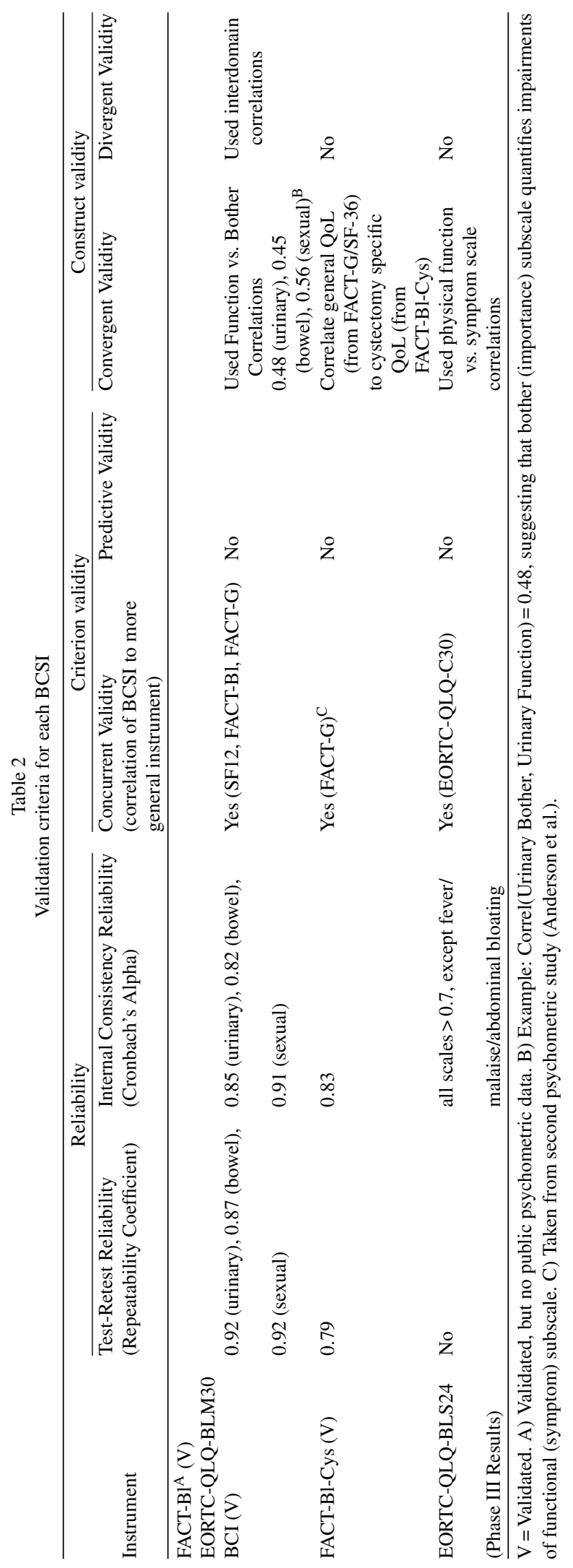


are actively used, a PubMed search using the terms "bladder cancer" and "quality of life" was performed, focusing on reviews. From there, our searches include the name of each instrument, including both the full name and commonly used abbreviations (e.g. "Functional Assessment of Bladder Cancer-Bladder Cystectomy" and "FACT-Bl-Cys"). A list of studies containing each instrument was compiled, with particular attention placed on the design of the study (retrospective vs. prospective), the study size and the statistical significance of the results (Supplementary data). This data was used to analyze the context in which each instrument has been utilized. Additionally, the studies which were used by the instrument makers to validate their respective instruments were analyzed (Tables 2 and 3).

\section{NON MUSCLE INVASIVE BLADDER CANCER INSTRUMENT}

There is only one HRQOL instrument that has been developed and validated for NMIBC and it is the:

\section{European Organization for Research and} Treatment of Cancer Quality of Life Core Questionnaire - Bladder Cancer Superficial (EORTC-QLQ-NMIBC24)

\section{Description and development}

The EORTC began developing quality of life instruments in 1986 with the EORTC C30 (a 30 item survey). This has formed the basis of numerous other disease specific HRQOL instruments. The EORTC implemented a modular design, in which disease-specific instruments are added to the core, cancer specific C30 in order to gauge HRQOL for specific types of cancer, and has developed a streamlined instruction manual to use with all EORTC instruments $[18,19]$. The additional instruments are most commonly validated through a standardized four phase process, including the generation of rel- evant issues, the creation of a provisional list of items, pre-testing and modification, and a large-scale international validation [20]. The EORTC-QLQNMIBC24 (NMIBC24) is a 24 item survey that gauges HRQOL for patients with intermediate to high risk NMIBC, including Ta, T1 and CIS. It is designed to be administered preoperatively (baseline), and at 2, 3, 6 and 12 month intervals. The NMIBC24 is the only instrument exclusively designed to compare HRQOL between nonmuscle-invasive treatment modalities, such as comparing 2 types of intravesical therapy. It should be noted, however, that the BCI (which will be discussed below) can be used to compare NMIBC treatments like intravesical therapy against other more invasive treatment modalities, like RC. The domains assessed in the NMIBC24 are listed in Table 1, and each item is scored out of 4 total points.

\section{Validation}

The NMIBC24 is currently in phase IV of the EORTC validation process [21]. It has been pretested (phase III), with validation data available from the pre-test (Table 2). It has not been tested in an international patient population, and it has not been gauged for test-retest reliability. A phase III trial included patients $(n=410)$ participating in the Bladder COX2 Inhibition Trial, which is a prospective, randomized study evaluating the addition of the COX inhibitor, celecoxib, to the standard treatment for NMIBC (Table 3) [22]. The study evaluated the scale, reliability, validity, responsiveness and acceptability of the NMIBC24.

Scaling was completed using multi-trait scaling analysis, in which questions were grouped into 6 domains with 5 additional items (Table 1). Internal consistency reliability was moderate to high in the domain items (Cronbach's $\alpha 0.49-0.90$ ), suggesting that the grouped domains adequately measured their intended symptoms. To test criterion validity, the researchers correlated scales of the $\mathrm{C} 30$ with those of the BLS24. Most correlations were low $(r<0.4)$,

Table 3

Characteristics of the validation studies for bladder cancer HRQOL instruments

\begin{tabular}{|c|c|c|c|c|c|}
\hline Instrument & $\begin{array}{l}\text { Sample } \\
\text { Size }\end{array}$ & $\begin{array}{l}\text { Randomized } \\
\text { treatment type? }\end{array}$ & Prospective/Retrospective & $\begin{array}{l}\text { Preoperative } \\
\text { survey administered? }\end{array}$ & Time Points Used \\
\hline \multicolumn{6}{|l|}{$\overline{\mathrm{FACT}-\mathrm{Bl}(\mathrm{V})^{\mathrm{A}}}$} \\
\hline \multicolumn{6}{|l|}{ EORTC-QLQ-BLM30 } \\
\hline BCI (V) & 315 & No & Retrospective & No & Variable \\
\hline FACT-B1-Cys $(\mathrm{V})^{\mathrm{B}}$ & 50,190 & No, No & Retrospective, Retrospective & No, Yes & $\begin{array}{l}>1 \text { year } / 4 \text { weeks later, } \\
\text { Preop } / 1 \text { year postop }\end{array}$ \\
\hline EORTC-QLQ-BLS24 & 410 & Yes & Prospective & Yes & preop, $2 \mathrm{~m}, 3 \mathrm{~m}, 6 \mathrm{~m}, 12 \mathrm{~m}$ \\
\hline
\end{tabular}

$V=$ validated. A) No psychometric data available. B) psychometric study 1 (Cookson et al.), psychometric study 2 (Anderson et al.) 
indicating that the core $\mathrm{C} 30$ and its NMIBC module have little overlap and are fit to be co-administered. Longitudinal data was calculated at baseline, 2, 3, 6 and 12 months. The NMIBC24 was found to be longitudinally responsive, with cognitive function, nausea and vomiting, dyspnea, and physical scores deteriorating over time. However, the NMIBC24's responsiveness, or ability to detect clinically meaningful differences between treatments, has not been definitively determined. Finally, acceptability was measured by calculating the completion rates of the surveys over the 12 month time interval. These rates ranged from $85.9 \%$ (6 months) to $94.3 \%$ (12 months).

\section{Summary}

The NMIBC24 is the only BCSI tested in a prospective, randomized trial (Table 3 ). Preliminary Phase III psychometric data suggests that it is a strong instrument, given its validity and responsiveness over time [22]. Additionally, its randomized design ensures that a patient's prior health status does not affect the choice of treatment [10]. Although phase III testing provided the NMIBC24 with some psychometric data, it lacks test-retest reliability and international testing data. The NMIBC24 cannot be fully validated until this information is made available after phase IV. Particularly, investigators should be wary when using a translated form of the survey, given that the instrument is not yet proven to be culturally adaptive. That being said, the EORTC has a robust process for preparing its surveys for international use, and once available, translated forms of the NMIBC24 will conform to the same stringent criteria as the original survey [20]. The responsiveness of the NMIBC24 is questioned as it has yet to show any statically significant differences over 3 studies in which it was used (Supplementary data) $[8,10,11,13]$. We recommend the NMIBC24 be used as a tool for investigators interested in comparing NMIBC treatments.

\section{MUSCLE INVASIVE BLADDER CANCER INSTRUMENTS}

\section{European Organization for Research and Treatment of Cancer Quality of Life Core Questionnaire - Bladder Cancer Muscle Invasive (EORTC-QLQ-BLM30)}

\section{Description and development}

The EORTC-QLQ-BLM30 (BLM30) is a nonvalidated 30 item survey that gauges HRQOL for patients with T2-T4 muscle-invasive bladder can- cer (MIBC). It includes questions that specifically address radical cystectomy (RC), such as assessing urostomy problems, catheter use and body image [21]. The BLM30 was created as a module to be used in conjunction with the $\mathrm{C} 30$, and as with all EORTC instruments, the BLM30 is validated via the EORTC's standardized four phase process [20]. Each item is scored on a 4 point scale, with a higher score reflecting a better HRQOL.

\section{Validation}

Although phase III testing can support limited psychometric data, none is publicly available for the BLM30, making it difficult to assess the validity and reliability of the instrument [21]. That being said, even without validation data the BLM30 has been utilized 9 times (Supplementary data). During phase IV, large scale, international field testing will provide information regarding validity, responsiveness, reliability, acceptability, and cross cultural adaptation.

\section{Summary}

The BLM30 is used to determine HRQOL in a broad context of situations. This is appealing for investigators who are interested in studying invasive procedures which affect multiple systems. For instance, two studies involving pelvic exenteration for rectal cancer include the BLM30, in addition to other EORTC instruments such as the core C30 and the CR38 (for colorectal cancer) [23, 24]. While most instruments typically compare two treatments, EORTC instruments (particularly the BLM30) were used to compare varying methods within a given treatment modality. For instance, Mischinger et al. used the BLM30 to compare HRQOL in orthotopic neobladder patients who received a $60 \mathrm{~cm}$ StuderPouch with those who received a $40 \mathrm{~cm}$ I-Pouch, with the I pouch patients scoring better in the continence domain [25].

Likely, the BLM30's widespread usage is a function of the variety of instruments offered by the EORTC, and the ease of scoring these instruments and interpreting data based on the same instruction manual [18]. A similar instrument is the FACTBl-Cys (described below), which can be used in conjunction with other FACT instruments to cover a wide symptom burden. Thus, the decision to use the BLM30 vs. the FACT-Bl-Cys in studying RC in MIBC patients is up to the investigator, with perhaps a slight advantage going to the FACT due to its validation. However, given the trend of its current usage 
(Supplementary data), the BLM30 should be considered for alternative situations other than comparing bladder cancer treatment modalities.

\section{Functional Assessment of Cancer Therapy - Bladder Cystectomy (FACT-Bl-Cys)}

\section{Description and development}

The FACT-Bl-Cys (formerly the FACT-VCI) is a validated questionnaire designed for patients undergoing RC and UD. It focuses on urinary, bowel, and sexual symptoms following cystectomy and is recommended to be administered in conjunction with the FACT-G; a 27 item questionnaire which is divided into physical, social/familial, emotional, and functional domains [14]. The FACT-Bl-Cys includes 17 items taken from 3 previously validated instruments (the FACT-Bl, the FACT-C for colorectal cancer and the FAIT-U for urinary incontinence) [26]. These items cover urinary (9 questions), bowel (2 questions), and sexual symptoms (1 question). Development of the instrument involved an expert panel meeting, a literature review and semi-structured group interviews with RC patients [26].

\section{Validation}

The FACT-B1-Cys was validated in a retrospective trial of 50 patients [26]. Each of the 17 items is scored on a 5 point Likert scale and added to form a single score with no subdomains. Internal consistency reliability was evaluated with a Cronbach's $\alpha$ of 0.83 , suggesting that the subscale questions were effective at measuring their intended symptoms. Construct validity was calculated by comparing the FACT-Bl-Cys with the more generic FACT-G and SF36. The results concluded that higher general quality of life scores from the SF36 and FACT-G correlate with a higher cystectomy-specific quality of life score from the FACT-Bl-Cys, as could be expected in an instrument which successfully measures HRQOL in bladder cancer. Additionally, test-retest reliability was assessed at 4 weeks, with a high correlation of 0.79. In a separate retrospective study of 190 patients, Anderson et al re-evaluates the psychometric properties of the FACT-Bl-Cys [27]. In this larger sample size, the validity of the previous psychometric data remained consistent. Additionally, this study showed the ability of the FACT-Bl-Cys to discriminate between HRQOL in ileal conduit (IC) patients and orthotopic neobladder (ONB) patients, with IC patients showing a higher HRQOL at postoperative year 1. This finding suggests that the FACT-Bl-Cys is able to detect valuable clinical information between UD types.

\section{Summary}

As the only instrument developed and validated particularly for the bowel, bladder, and sexual concerns of the RC population, the FACT-Bl-Cys is an excellent tool for measuring HRQOL in these patients. It covers both systemic (FACT-G) and bladder cancer specific symptoms, and it has a proven ability to discriminate between UD types. However, it may not be optimal for studying HRQOL outcomes within a particular diversion type as there is a limited scope regarding post cystectomy management of urinary diversion. Other limitations in the Fact-Bl-Cys include small sample size and only two time points evaluating longitudinal responsiveness.

\section{Conclusion for Muscle Invasive Bladder Cancer Instruments}

There are two instruments that have been developed to assess HRQOL for muscle invasive bladder cancer: the BLM 30 and the FACT-B1-Cys. Although the BLM30 also contains questions pertaining to $\mathrm{RC}$ with UD, we favor the FACT-B1-Cys due to its validation. In accordance with its design, we suggest that investigators use the FACT-Bl-Cys to study HRQOL in patients with RC and UD.

\section{INCLUSIVE BLADDER CANCER INSTRUMENTS}

\section{Functional Assessment of Cancer Therapy - Bladder (FACT-Bl)}

\section{Description and development}

The FACT-Bl is a validated questionnaire designed for patients with bladder cancer, including MIBC and NMIBC. It consists of a general version (FACT$\mathrm{G})$ to which 12 questions of the bladder-specific scale are added. The bladder-specific answers are totaled, and added to the FACT-G score, with a higher score reflecting better HRQOL. These questions cover urinary function, bowel function, sexual function, body image, appetite and ostomy care (only answered by patients with ostomy) [28]. The FACTBl was designed by the Functional Assessment of Chronic Illness Therapy (FACIT) Measurement System, which has been developing questionnaires since 1987, beginning with the core FACT-G [15]. For all of its instruments, FACIT implements a four phase development and validation procedure consisting of 
item generation, item reduction, scale construction and psychometric evaluation [29].

\section{Validation}

Although no psychometric data specific to the FACT-Bl is publicly available, the FACT-Bl has demonstrated reliability, validity and sensitivity to change over time. Each newly constructed FACIT subscale undergoes an initial assessment of validity using a sample of at least 50 patients [29]. The validation typically involves patient completion of a baseline assessment, a test-retest assessment 3 to 7 days later and a third assessment 2 to 3 months later to demonstrate sensitivity to change over time. Relevant sociodemographic and treatment data is also collected, and a host of other surveys are administered at the baseline period and at the 2 to 3 month retest to determine convergent and divergent validity. A comprehensive analysis of the data gathered yields useful psychometric information and establishes initial reliability and validity of the scale.

\section{Summary}

The FACT-Bl is a reliable, validated, and responsive instrument which was designed for use across the range of bladder cancer treatments and stages. It differs from the EORTC instruments in that it is validated, and that it was designed to compare HRQOL between MIBC and NMIBC patients [30-32]. Thus, the FACT-Bl can be administered by investigators comparing two UD types, two intravesical treatments, or comparing $\mathrm{RC}$ to bladder-sparing treatments. The FACT-Bl is much the same as the BCI (described below), which was also designed to be used across treatment modalities and diseases [33]. Unlike the BCI, the FACT-Bl lacks the ability to discriminate which symptoms are most disturbing to the patient, because it does not include separate function and bother subscales. Additionally, the FACT-Bl does not break its questions into separate domains (for example, bowel, bladder and sexual symptoms) as does the BCI. These separate domains aid the investigator in creating a more holistic view of the patient experience [33].

\section{Bladder Cancer Index (BCI)}

\section{Description and development}

The University of Michigan's Bladder Cancer Index (BCI) was designed as an all-inclusive instrument for patients with nonmuscle-invasive and muscle-invasive disease of any stage. Each of its three domains has a function and bother subscale component and all domains are scored separately using a 100 point Likert scale. During its development, items were first generated by an expert panel, then revised with the input of 62 bladder cancer survivors and finally pilot-tested in a separate group of 72 bladder cancer patients [33]. The final validated instrument contains 36 items divided into 3 domains (urinary, bowel and sexual). In terms of usage, the BCI is similar to the FACT-B1. Both instruments were designed for use across a range of diseases and treatments

\section{Validation}

Validation of the BCI included a group of 315 bladder cancer patients [33]. The 36 total items were placed into urinary, bowel and bladder domains using a sorting method called varimax rotation. Internal consistency reliability was high (Cronbach's $\alpha$ 0.77-0.94), suggesting that each domain measured its intended symptoms. External reliability was also high, with test-retest correlations of 0.92 (urinary), 0.87 (bowel) and 0.92 (sexual) over a 2 to 4 week period. Convergent and divergent validity revealed moderate intra-domain and weak inter-domain correlation respectively. This moderate convergent validity suggests that the bother subscale is an effective means of quantifying the function subscale, while the low divergent validity suggests that each of the three domains succeeds in measuring separate groups of symptoms. Concurrent validity was tested using three other relevant instruments: the SF-12, the FACT-G and the FACT-B1. Several components of the BCI subscales correlated well with the cancer-specific tools like the FACT-Bl and certain domains of the FACT$\mathrm{G}$, but less so with the non-cancer specific SF-12 $(r=0.07-0.34)$. This low correlation suggests that little overlap exists between the BCI and more general instruments. In a separate paper, Gilbert et al found the BCI to successfully detect differences in bother scores between cystectomy and native bladder treatment groups, suggesting responsiveness between treatment modalities [34].

\section{Summary}

The BCI is a strong instrument designed specifically for comparison across a range of disease stages and treatment modalities. Its bother subscale provides an unmatched quantitative measure of the severity of symptoms for urinary, bowel, and sexual domains. However, if the function and bother scores for a particular domain are different, it may be difficult for the investigator to determine which score should 


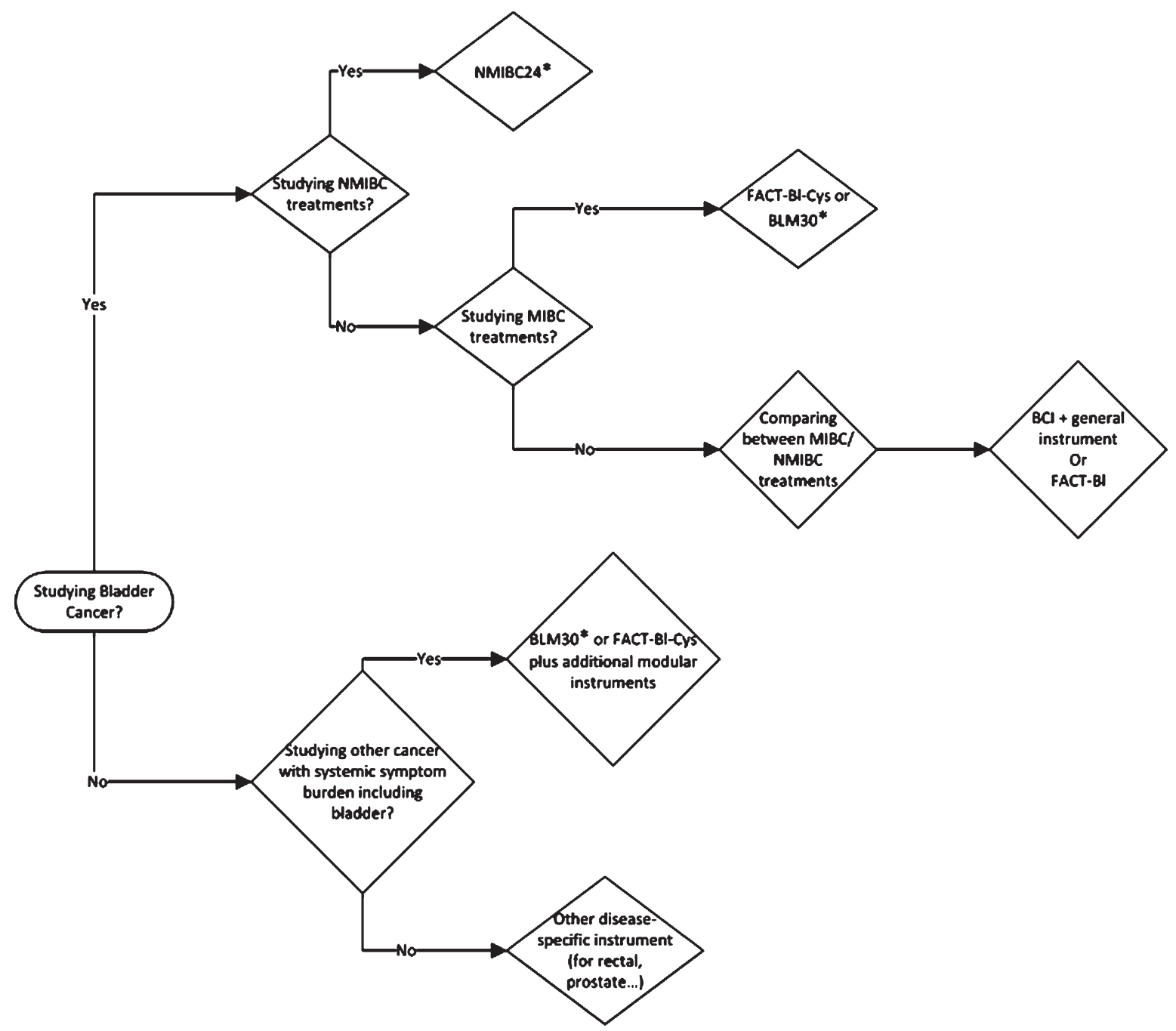

Fig. 1. Decision tree for instrument selection. *Non-validated.

be used to determine HRQOL. Discrepancies place a burden on the investigator to decide whether the patient's symptoms (the function score), or the degree of importance that the patient places on his or her symptoms (the bother score) should be used to determine HRQOL [34]. The BCI's main limitation is its low correlation to more general instruments. Thus, to include more systemic HRQOL data, researchers should consider administering a general instrument such as the SF-12, or a cancer specific tool such as the FACT-G. Additionally, the BCI's neutral design should be taken into account by researchers studying one specific treatment modality or studying genderspecific symptoms. For example, although questions involving erectile dysfunction are certainly not gender neutral, they provide key sexual information in evaluating a male patient's HRQOL. By the same token, questions regarding hardware specific for a non-continent UD do not apply to a patient with ONB, but are certainly crucial in evaluating the HRQOL of a patient with IC. As a final limitation, the BCI was validated in a cross sectional study and lacks significant longitudinal data and body image items.

\section{Conclusion for Inclusive Bladder Cancer Instruments}

We recommend investigators consider the BCI (coadministered with a general instrument and body image instrument) along with the FACT-Bl as tools for comparing MIBC with NMIBC treatments. These instruments are also preferred for patients with MIBC who are receiving treatment other than $\mathrm{RC}$, as they 
more thoroughly cover the concerns associated with a wider range of treatments. Additionally, we recommend the BCI to clinicians interested in studying the emphasis that patients place on the symptoms of bladder cancer, given the BCI's unique bother scale.

\section{CONCLUSIONS}

An investigator's choice of instrument(s) plays a substantial role in determining the quality of HRQOL data which is ultimately collected. It is necessary to collect data that covers both the overall well-being of the patient, as well as disease-specific concerns. Thus, investigators need to administer BCSIs along with a more general instrument. A process which we follow for deciding upon the appropriate instrument is outlined in Fig. 1. In the case of NMIBC, the investigator should utilize the non-validated NMIBC24, which is the only instrument designed to study HRQOL in patients receiving intravesical treatments. For MIBC, investigators have the option to use either the nonvalidated BLM30 or the validated FACT-B1-Cys. These instruments are accompanied with a broader questionnaire to more appropriately cover the concerns seen in MIBC patients. Both instruments are modular and extensively used, but the BLM30 is used in a wider range of contexts - particularly by investigators interested in urinary complications secondary to other cancer types, like rectal cancer. Finally, for investigators interested in comparing between MIBC and NMIBC treatment modalities, both the FACT$\mathrm{Bl}$ and the $\mathrm{BCI}$ are viable instruments. The $\mathrm{BCI}$ is non-modular, gender/UD-neutral, and should be administered with a more general instrument like the FACT-G, C30, or SF-36. In comparison, the FACT$\mathrm{Bl}$ is a modular instrument which is co-administered with the FACT-G. With the correct instrument in use, investigators can glean relevant HRQOL information that will inform clinical decision making and aid in counseling patients to live with their treatment course.

\section{CONFLICT OF INTEREST}

The authors have no conflict of interest to report.

\section{SUPPLEMENTARY MATERIAL}

The supplementary table is available in the electronic version of this article: http://dx.doi.org/ 10.3233/BLC-160057.

\section{REFERENCES}

[1] Ying J, Wang CJ, Yan J, Liauw SL, Straka C, Pistenmaa D, et al. Long-term Outcome of Prostate Cancer Patients Who Exhibit Biochemical Failure Despite Salvage Radiation Therapy After Radical Prostatectomy. Am J Clin Oncol [Internet]. 2015 Jul 9 [cited 2016 Jun 13]; Available from: http://www.ncbi.nlm.nih.gov/pubmed/26165416

[2] Riley GF, Potosky AL, Lubitz JD, Kessler LG. Medicare payments from diagnosis to death for elderly cancer patients by stage at diagnosis. Med Care [Internet]. 1995 Aug [cited 2015 Aug 5];33(8):828-41. Available from: http://www.ncbi.nlm.nih.gov/pubmed/7637404

[3] Patient Reported Outcomes (PROs) in Performance Measurement. Natl Qual Forum. 2013;

[4] Crossing the Quality Chasm: A New Health System for the 21st Century - PubMed - NCBI [Internet]. [cited 2015 Aug 5]. Available from: http://www.ncbi.nlm.nih.gov/ pubmed/25057539

[5] Wu AW, Hopkins J, Jensen RE, Salzberg C, Snyder C. Advances in the Use of Patient Reported Outcome Measures in Electronic Health Records Advances in the Use of Patient Reported Outcome Measures in Electronic Health Records 1. 2013;

[6] DSMICA. Guidance for Industry Patient-Reported Outcome Measures: Use in Medical Product Development to Support Labeling Claims Clinical/Medical Guidance for Industry Patient-Reported Outcome Measures: Use in Medical Product Development to Support Labeling Claims. 2009;301-443. Available from: http://www.fda.gov/Drugs/ GuidanceComplianceRegulatoryInformation/Guidances/ default.htm

[7] Patrick DL, Burke LB, Powers JH, Scott JA, Rock EP, Dawisha $\mathrm{S}$, et al. Patient-reported outcomes to support medical product labeling claims: FDA perspective. Value Health [Internet]. [cited 2016 Jun 13];10 Suppl 2:S125-37. Available from: http://www.ncbi.nlm.nih.gov/pubmed/17995471

[8] Wright JL, Porter MP. Quality-of-life assessment in patients with bladder cancer. Nat Clin Pract Urol [Internet]. 2007 Mar [cited 2016 Jun 13];4(3):147-54. Available from: http:// www.ncbi.nlm.nih.gov/pubmed/17347659

[9] Patrick DL, Erickson P. Health status and health policy: Quality of life in health care evaluation and resource allocation. Oxford University Press; 1993.

[10] Porter MP, Penson DF. Health related quality of life after radical cystectomy and urinary diversion for bladder cancer: A systematic review and critical analysis of the literature. J Urol [Internet]. 2005 Apr [cited 2016 Jun 13];173(4):1318-22. Available from: http://www. ncbi.nlm.nih.gov/pubmed/15758789

[11] Gerharz EW, Månsson A, Månsson W. Quality of life in patients with bladder cancer. Urol Oncol [Internet]. [cited 2016 Jun 13];23(3):201-7. Available from: http://www.ncbi.nlm.nih.gov/pubmed/15907722

[12] Ware JE, Kristin Snow with K, Mark Kosinski M, Barbara Gandek M. SF-36 Health Survey Manual and Interpretation Guide.

[13] Parkinson JP, Konety BR. Health related quality of life assessments for patients with bladder cancer. J Urol [Internet]. 2004 Dec [cited 2016 Jun 13];172(6 Pt 1):2130-6. Available from: http://www.ncbi.nlm.nih.gov/ pubmed/15538218

[14] Aaronson NK, Ahmedzai S, Bergman B, Bullinger M, Cull A, Duez NJ, et al. The European Organization for Research and Treatment of Cancer QLQ-C30: A quality-of-life 
instrument for use in international clinical trials in oncology. J Natl Cancer Inst [Internet]. 1993 Mar 3 [cited 2016 Jun 13];85(5):365-76. Available from: http://www.ncbi. nlm.nih.gov/pubmed/8433390

[15] Cella DF, Tulsky DS, Gray G, Sarafian B, Linn E, Bonomi A, et al. The Functional Assessment of Cancer Therapy scale: Development and validation of the general measure. J Clin Oncol [Internet]. 1993 Mar [cited 2016 Jun 13];11(3):570-9. Available from: http://www.ncbi. nlm.nih.gov/pubmed/8445433

[16] Hopwood P, Fletcher I, Lee A, Al Ghazal S. A body image scale for use with cancer patients. Eur J Cancer [Internet]. 2001 Jan [cited 2016 Jun 13];37(2):189-97. Available from: http://www.ncbi.nlm.nih.gov/pubmed/11166145

[17] Aaronson N, Alonso J, Burnam A, Lohr KN, Patrick DL, Perrin E, et al. Assessing health status and quality-oflife instruments: Attributes and review criteria. Qual Life Res [Internet]. 2002 May [cited 2016 Jun 13];11(3):193205. Available from: http://www.ncbi.nlm.nih.gov/pubmed/ 12074258

[18] EORTC Quality of Life Group. Guidelines for assessing quality of life in EORTC clinical trials. 2002; Available from: http://groups.eortc.be/qol/sites/default/xfiles/ archives/clinical_trials_guidelines_qol.pdf

[19] Bergman B, Aaronson NK, Ahmedzai S, Kaasa S, Sullivan M. The EORTC QLQ-LC13: A modular supplement to the EORTC Core Quality of Life Questionnaire (QLQ-C30) for use in lung cancer clinical trials. EORTC Study Group on Quality of Life. Eur J Cancer [Internet]. 1994 [cited 2016 Jun 13];30A(5):635-42. Available from: http://www.ncbi. nlm.nih.gov/pubmed/8080679

[20] Johnson C, Aaronson N, Blazeby JM, Bottomley A, Fayers $\mathrm{P}$, Koller M, et al. EORTC QUALITY OF LIFE GROUP Guidelines for Developing Questionnaire Modules. 2011;

[21] Bladder Cancer: EORTC QLQ-NMIBC24, EORTC QLQBLM30 [Internet]. Available from: http://groups.eortc.be/ qol/bladder-cancer-eortc-qlq-nmibc24-eortc-qlq-blm30

[22] Blazeby JM, Hall E, Aaronson NK, Lloyd L, Waters R, Kelly JD, et al. Validation and reliability testing of the EORTC QLQ-NMIBC24 questionnaire module to assess patient-reported outcomes in non-muscle-invasive bladder cancer. Eur Urol [Internet]. 2014 Dec [cited 2016 Jun 13];66(6):1148-56. Available from: http://www.ncbi.nlm. nih.gov/pubmed/24612661

[23] Guren MG, Wiig JN, Dueland S, Tveit KM, Fosså SD, Waehre $\mathrm{H}$, et al. Quality of life in patients with urinary diversion after operation for locally advanced rectal cancer. Eur J Surg Oncol [Internet]. 2001 Nov [cited 2016 Jun 13];27(7):645-51. Available from: http://www.ncbi. nlm.nih.gov/pubmed/11669593

[24] Rezk YA, Hurley KE, Carter J, Dao F, Bochner BH, Aubey JJ, et al. A prospective study of quality of life in patients undergoing pelvic exenteration: Interim results. Gynecol Oncol [Internet]. 2013 Feb [cited 2016 Jun 13];128(2):191-7. Available from: http://www.ncbi.nlm. nih.gov/pubmed/23063761

[25] Mischinger J, Abdelhafez MF, Todenhöfer T, Schwentner C, Aufderklamm S, Stenzl A, et al. Quality of life outcomes after radical cystectomy: Long-term standardized assessment of Studer Pouch versus I-Pouch. World J Urol [Internet]. 2015 Oct [cited 2016 Jun 13];33(10):1381-7. Available from: http://www.ncbi.nlm.nih.gov/pubmed/25501497

[26] Cookson MS, Dutta SC, Chang SS, Clark T, Smith JA, Wells N. Health related quality of life in patients treated with radical cystectomy and urinary diversion for urothelial carcinoma of the bladder: Development and validation of a new disease specific questionnaire. J Urol [Internet]. 2003 Nov [cited 2016 Jun 13];170(5):1926-30. Available from: http://www.ncbi.nlm.nih.gov/pubmed/14532809

[27] Anderson CB, Feurer ID, Large MC, Steinberg GD, Barocas DA, Cookson MS, et al. Psychometric characteristics of a condition-specific, health-related quality-of-life survey: The FACT-Vanderbilt Cystectomy Index. Urology [Internet]. 2012 Jul [cited 2016 Jun 13];80(1):77-83. Available from: http://www.ncbi.nlm.nih.gov/pubmed/22608798

[28] Chin JL, Izawa JI. Treatment of radiation failure in prostate cancer. Can J Urol [Internet]. 2006 Apr [cited 2016 Jun 13];13 Suppl 2:48-51. Available from: http://www.ncbi. nlm.nih.gov/pubmed/16672129

[29] Webster K, Cella D, Yost K. The Functional Assessment of Chronic Illness Therapy (FACIT) Measurement System: Properties, applications, and interpretation. Health Qual Life Outcomes [Internet]. 2003 [cited 2016 Jun 13];1:79. Available from: http://www.ncbi. nlm.nih.gov/pubmed/14678568

[30] Karvinen KH, Courneya KS, North S, Venner P. Associations between exercise and quality of life in bladder cancer survivors: A population-based study. Cancer Epidemiol Biomarkers Prev [Internet]. 2007 May [cited 2016 Jun 13];16(5):984-90. Available from: http://www. ncbi.nlm.nih.gov/pubmed/17507626

[31] Botteman MF, Pashos CL, Hauser RS, Laskin BL, Redaelli A. Quality of life aspects of bladder cancer: A review of the literature. Qual Life Res [Internet]. 2003 Sep [cited 2016 Jun 13];12(6):675-88. Available from: http://www.ncbi.nlm.nih.gov/pubmed/14516177

[32] Månsson $\mathrm{A}$, Davidsson $\mathrm{T}$, Hunt $\mathrm{S}$, Månsson W. The quality of life in men after radical cystectomy with a continent cutaneous diversion or orthotopic bladder substitution: Is there a difference? BJU Int [Internet]. 2002 Sep [cited 2016 Jun 13];90(4):386-90. Available from: http://www.ncbi.nlm.nih.gov/pubmed/12175394

[33] Gilbert SM, Dunn RL, Hollenbeck BK, Montie JE, Lee CT, Wood DP, et al. Development and validation of the Bladder Cancer Index: A comprehensive, disease specific measure of health related quality of life in patients with localized bladder cancer. J Urol [Internet]. 2010 May [cited 2016 Jun 13];183(5):1764-9. Available from: http://www.ncbi.nlm.nih.gov/pubmed/20299056

[34] Gilbert SM, Wood DP, Dunn RL, Weizer AZ, Lee CT, Montie JE, et al. Measuring health-related quality of life outcomes in bladder cancer patients using the Bladder Cancer Index (BCI). Cancer [Internet]. 2007 May 1 [cited 2016 Jun 13];109(9):1756-62. Available from: http://www.ncbi.nlm.nih.gov/pubmed/17366596 


\section{APPENDIX 1: INSTRUMENT VALIDATION/RELIABILITY MEASURE DEFINITIONS [13]}

Validity - How well an instrument measures its intended target (the general term "validity" is often used interchangeably with the specific term "construct validity," although some studies only utilize criterion validity to establish the validity of the instrument)

1. Criterion Validity - How well a variable predicts an outcome based on information from other variables

a. Concurrent Validity - Correlation of instrument scores with scores from other established tests (ex. correlating FACT-B1Cys score with scores from the FACT-G)

b. Predictive Validity - Correlation of instrument scores and future measurable outcomes (not widely used in bladder cancer studies to show criterion validity)

2. Construct Validity - The meaningfulness of an instrument (often determined definitively after the instrument has been used for many years however, convergent and divergent validity can be considered reflective of construct validity)

a. Convergent Validity - Do different constructs measuring the same variable converge on the same result? In other words, do two different traits that the instrument sets out to measure correlate closely enough? (ex. does the urinary function subscale of the BCI correlate with the urinary bother subscale of the BCI?)

b. Divergent Validity - Is the construct you are interested in different from other constructs in your study? In other words, are two different traits that the instrument sets out to measure sufficiently different? (ex. does the urinary function subscale of the BCI correlate too closely to the sexual bother subscale of the BCI?)

Note: A moderate level of both convergent and divergent validity is desired to suggest that the instrument has construct validity

Test-Retest Reliability - The variation in measurements of a test or test item taken by the same person at two points in time (typically 2-4 weeks apart).

Internal Consistency Reliability - Do the items on a test, which are all supposed to measure the HRQOL, produce similar scores? To find this, correlate individual item scores to the overall test score using Cronbach's alpha. If Cronbach's alpha is too high, likely the questions are redundant. If it is too low, the questions are unrelated to measuring the concept of HRQoL.

Responsiveness - Can the instrument detect clinically meaningful differences between treatments? In other words, do the instrument's scores between differing treatments show no variance at all (not responsive), or too much variance (too responsive)?

Longitudinal Responsiveness - Is the instrument sensitive to change over time? For instance, is there a difference between preoperative scores and scores at 2 months, 3 months, 6 months and 1 year?

Acceptability - Is the instrument easy for the respondent to understand and to complete in a timely manner?

Cultural Adaptation-Does the instrument maintain its integrity in other languages?

Principle Component Analysis - Should items be broken into subscales, each having an individual score? Or should all items be part of the same single summary scale having just one score? 